Research Paper

\title{
Jak2a regulates erythroid and myeloid hematopoiesis during zebrafish embryogenesis
}

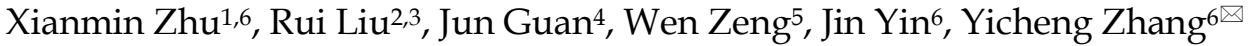 \\ 1. Department of Medical Oncology, Hubei Cancer Hospital, Wuhan, Hubei 430079, China. \\ 2. Department of Histology and Embryology, School of Basic Medical Science, Wuhan University, Wuhan, Hubei 430071, China. \\ 3. Department of Anatomy, Hubei University of Medicine, Shiyan, Hubei442000, China. \\ 4. Department of Hematology, Wuhan integrated TCM \& Western Medicine hospital, Wuhan, Hubei 430030, China. \\ 5. Department of Ophthalmology, Zhongnan Hospital of Wuhan University, Wuhan, Hubei 430030, China. \\ 6. Department of Hematology, Tongji Hospital, Tongji Medical College, Huazhong University of Science and Technology, Wuhan, Hubei 430030, China. \\ $\square$ Corresponding author: Yicheng Zhang, E-mail address: yczhang@tih.tjmu.edu.cn \\ (c) Ivyspring International Publisher. This is an open access article distributed under the terms of the Creative Commons Attribution (CC BY-NC) license \\ (https://creativecommons.org/licenses/by-nc/4.0/). See http://ivyspring.com/terms for full terms and conditions.
}

Received: 2016.12.24; Accepted: 2017.04.21; Published: 2017.07.18

\begin{abstract}
Zebrafish (Danio rerio) are an attractive vertebrate model for the molecular dissection of disease mechanisms. Janus kinase (JAK)/signal transducer and activator of transcription (stat) has been defined through studies of cytokine signaling pathways in mammals. Here, we examined the expression level of Jak2a, which is a homolog of mammalian jak2 in zebrafish, by quantitative reverse transcriptase (RT)-PCR, and the peak of mRNA expression occurred at 3.75 hours post fertilization (hpf). The overexpression of Jak2a was proven by real-time Q-PCR and Western blot in 1-4-cell stage embryos injected with $400 \mathrm{ng} / \mu$ l full-length jak2a mRNA as well as gfil.1, gatal, mpo and $\beta$-embryonic hemoglobin as detected by real-time Q-PCR. Moreover, jak2a mRNA significantly increased the $\mathrm{GFP}^{+}$population in the transgenic zebrafish lines $\mathrm{Tg}$ (gatal: $g \mathrm{fP}$ ) (uninjected embryos: $17.22 \pm 1.70 \%$; embryos injected with jak2a mRNA: $21.31 \pm 2.11 \%, p<0.01$ ) and $\mathrm{Tg}$ (mpo: $g f$ ) (uninjected embryos: 3.86 \pm 1.94 ; embryos injected with jak2a mRNA: $6.64 \pm 1.30 \%$, $\mathrm{p}<0.01$ ) compared with the control group. Thus, our data indicate that Jak2a plays an important role in erythropoiesis and myeloid hematopoiesis.
\end{abstract}

Key words: zebrafish, Jak2a, mRNA, overexpression, hematopoiesis

\section{Introduction}

Recently, the discovery of an acquired somatic mutation in the JAK2 gene resulting in a Val-to-Phe substitution at position 617 (JAK2 ${ }^{\text {V617F }}$ ) was described and further revised our molecular cognition regarding myeloproliferative neoplasia (MPN) [1-7]. In mammals, the JAK family consists of 4 members that include JAK1, JAK2, JAK3 and TYK2, and JAK2 is thought to transduce the signals of hematopoietic cytokines to enhance erythroid cell proliferation [8]. Jak2a was first proposed in the Andrew $C$ group based on sequence comparison of the PCR product with JAK-directed degenerate primer pairs. Jak2a exhibits a high degree of sequence conservation, and the protein has an open reading frame with high similarity to the mammalian JAK2 proteins from mice, humans, rats and pigs based on conceptual translation
[9]. Due to the embryonic lethal nature of homozygous Jak2null mice, the role of Jak2a in embryonic hematopoiesis remained unknown until $2007[10,11]$.

Because the species hematopoiesis can be visualized in vitro with transgenic fluorescent protein reporters, zebrafish are considered to be an ideal model organism for the study of hematopoiesis during embryonic development and neoplastic transformation $[12,13]$. In zebrafish, jak2a is known to be primarily expressed in the erythropoietic system, whereas jak $2 \mathrm{~b}$ is expressed in the developing eyes and pronephric ducts [14]. The expression of jak2a was first examined using microarray analysis in the zebrafish chordin morphant, and its upregulation was detected in the intermediate cell mass (ICM) and 
characterized by the expansion of primitive hematopoiesis [15]. Furthermore, the role of jak2a in hematopoiesis was subsequently studied with qualitative and quantitative analyses using morpholino (MO) knockdown, which revealed that erythrocytosis in zebrafish embryos is mediated by jak2a activation [9]. Subsequently, jak2aV581F, which is an ortholog of the human JAK2 ${ }^{\text {V617F }}$ mutation, was generated by the Anskar group, and its expression induces a significant increase in erythropoiesis and a downregulation of erythropoietin expression [16].

In this study, we examined the expression of jak2a during the embryonic development of zebrafish and investigated its significant role in erythroid and myeloid hematopoiesis by microinjecting full-length jak2a mRNA into the embryos. Furthermore, the differences between and concrete mechanisms of the jak2a group and jak2aV581F in erythroid and myeloid hematopoiesis should be further explored in the future.

\section{Methods}

\section{Zebrafish}

Wild-type zebrafish and transgenic zebrafish lines Tg (gata1: $g f p$ and mpo: $g f p$ ) were obtained from the Institute of Hydrobiology of the Chinese Academy of Science and raised under standard conditions at $28.5^{\circ} \mathrm{C}$ in a recirculating aquaculture system at Tongji Medical College.

The full-length zebrafish jak2a coding sequence was cloned into the pGM-T vector, and the following primers were used to amplify jak2a by reverse transcription PCR:

sense 5'- CCGGAATTC GCTTTGAGTCGGTT

TCTCCGGTTCC,

antisense 3'-TGCTCTAGA CCTCATGCAGTCG CTGAATAAGTCC.

The pGM-T vector containing the jak2a insert was linearized with $\mathrm{NdeI}$ and transcribed in vitro from the $T 7$ promoter with a mMessage mMachine Kit (Ambion, Austin, TX, USA).

\section{Modulation of jak2a}

To study the activity of jak2a in zebrafish, the full-length mRNA of zebrafish jak2a was injected into one- to four-cell stage embryos to establish the overexpression model of jak2a. The control group was established from the same untreated embryos. The injected embryos were raised under standard conditions at $28.5^{\circ} \mathrm{C}$ and observed every four hours.

\section{Reverse transcription and quantitative real-time PCR}

Total mRNA was extracted from 1-dpf embryos with TRIzol according to the manufacturer's instructions. Real-time Q-PCR was performed on an ABI Prism 7700 Sequence Detector (Applied Biosystems Inc., Foster City, CA, USA), and the relative expressions of the genes for jak2a, gfi1.1, erythroid (gata1, $\beta$-embryonic hemoglobin), and heterophilic granulocyte (mpo) were examined. The analysis was performed with the 2- C (T) method as described previously [17].

\section{Flow cytometric analysis of erythropoiesis}

Pools of 20-30 embryos were dechorionated and digested with trypsin for 5 minutes at room temperature. Next, single-cell suspensions were obtained via pipetting and sequential passing through $40-\mu \mathrm{m}$ cell strainers. The cells were washed and re-suspended in phosphate-buffered saline before examination by flow cytometry.

\section{RNA whole-mount in situ hybridization and hemoglobin staining}

Digoxigenin-UTP (Roche)-labeled antisense RNA probes were generated by linearizing the pGM-T vector using ApaI and in vitro transcription with SP6 polymerase. Whole-mount in situ hybridization was performed as previously described [18]. Two-day post-fertilization embryos were stained with O-dianisidine (Sigma) for analysis of the hemoglobinized cells [18].

\section{Western blot analysis}

The embryos were dechorionated and lysed in RIPM buffer supplemented with protease inhibitor cocktail and $\beta$-glycerophosphate. Then, the proteins were separated by gel electrophoresis, electrotransferred to PVDF membranes and incubated in a solution containing the following antibodies: anti- $\beta$ actin antibody, jak2 rabbit $\mathrm{mAb}$ and gfi1 rabbit $\mathrm{mAb}$ (both from Santa Cruz, USA). The signals were detected with a BCIP/NBT system (Sigma, MO, USA).

\section{Image acquisition and analysis}

Transgenic Tg (gata1: $g f p$ ) and Tg (mpo: $g f p$ ) embryos and larvae were observed under an Olympus fluorescence microscope for the detection of GFP. Data from at least three independent experiments are displayed as the relative means \pm the standard errors of the mean and were examined with the Student's $t$-test. SPSS v11.5 was used for the statistical analyses.

\section{Results}

\section{Expression of jak2a in zebrafish}

The RNA of the developmental series of zebrafish embryos and adult tissues was extracted for the evaluation of the expression levels of jak $2 a$ by 
quantitative reverse transcriptase (RT) PCR. Zebrafish jak2a was detected from the 1-cell stage to $24 \mathrm{hpf}$, and the maternal transcripts for zebrafish jak2a were proven to be present (Figure 1). The expression peak was detected at $3.75 \mathrm{hpf}$, after which the levels gradually decreased until $12 \mathrm{hpf}$, and the copies were continuingly detectable until $96 \mathrm{hpf}$. In the adult zebrafish, 15 tissues were used to detect the expression of jak2a, and nearly all were confirmed to express this RNA (Figure 2). High levels of jak2a were detected in the muscle, fin and skin, whereas the ovary, testis and spleen exhibited low levels of expression. The expression levels in other tissues, such as the kidney, cheek, gallbladder, eye, swim bladder, brain, intestine, liver, and heart, were between these two groups. The kidneys are the hematogenic organs in adult zebrafish, and the expression levels of jak2a in the kidneys were not very high.

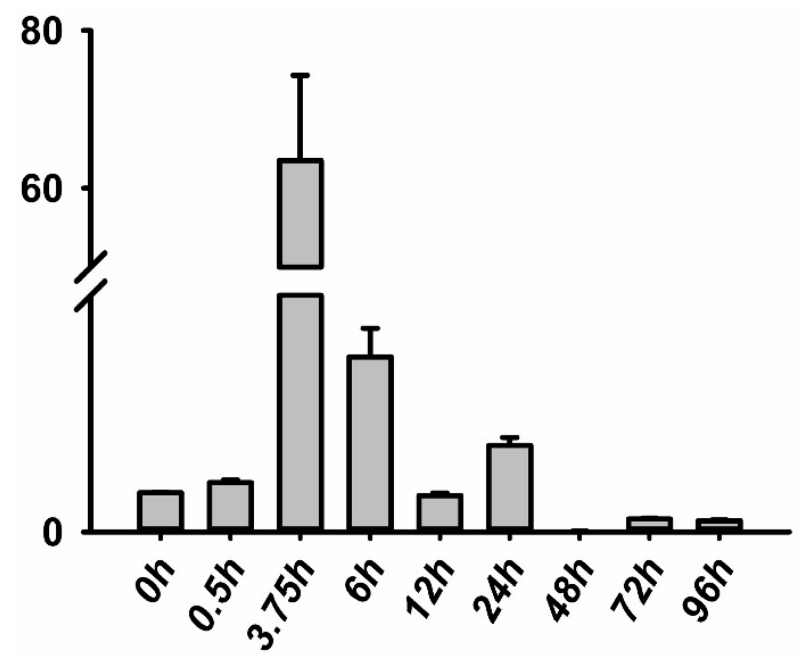

Figure 1. Expression level of the jak2a gene in zebrafish. RT-PCR analysis revealing the expression level of jak2a during embryogenesis.

\section{Expression of jak $2 a$ increased in the jak2a-overexpression group compared with the controls}

To overexpress jak2a in the zebrafish, we microinjected 2 ng of full-length jak2a mRNA with T7 polymerase per embryo. T7 polymerase initiates transcription at a $\mathrm{T} 7$ promoter, and the in vitro jak2a mRNA product was proven to be consistent with the expected results by gel electrophoresis (Figure 3). Jak2a was injected at the concentration of $400 \mathrm{ng} / \mu 1$ because a dose-finding study revealed that the injection of higher concentrations of jak2a mRNA was associated with toxicity and mortality. All embryos were microinjected at the 1-4 cell stage.

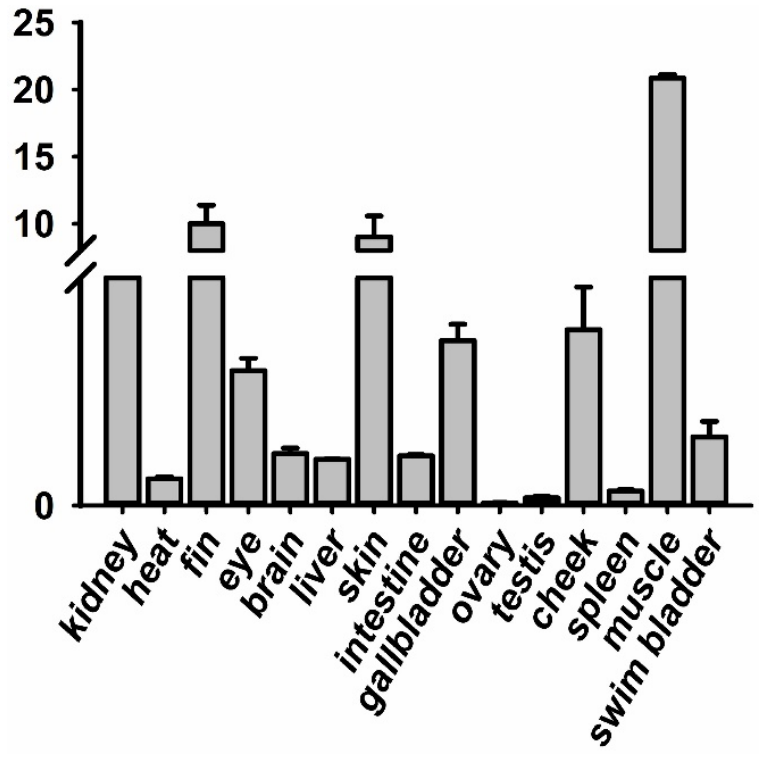

Figure 2. RT-PCR analysis revealing the expression level of jak2a during the development of adult zebrafish tissues.

\section{1}

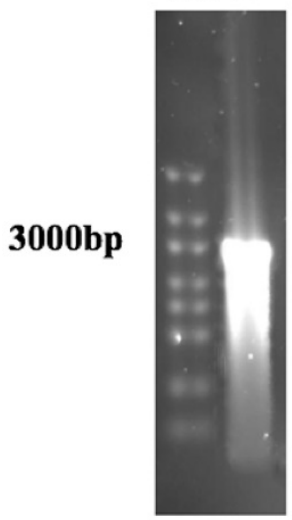

Figure 3. The establishment of the overexpression of jak2a mRNA model in zebrafish. Examination of the jak2a mRNA by gel electrophoresis.

To determine whether the overexpression jak2a mRNA in vivo was successful, real-time Q-PCR was performed on the overexpressing embryos at the molecular level, and Western blot analysis was applied to examine the protein level. As illustrated in Figure 4, the expression of jak2a in the injected $1 \mathrm{dpf}$ embryos was significantly upregulated by 4.5 -fold and accompanied increases in the expressions of gfi1, gata1, mpo and $\beta$-embryonic hemoglobin of 3.0-, 2.0-, 3.0- and 1.0-fold, respectively, compared with the wild-type group.

Furthermore, Western blot analysis using Jak2 and Gfi1 antibodies was applied to the protein extracts from the embryos that were injected with 400 $\mathrm{ng} / \mu \mathrm{l}$ jak2a. The results revealed that very low levels of Jak2a protein were observed in the control group compared with the overexpression group, and similar results were observed for the Gfi1 protein (Figure 5). 


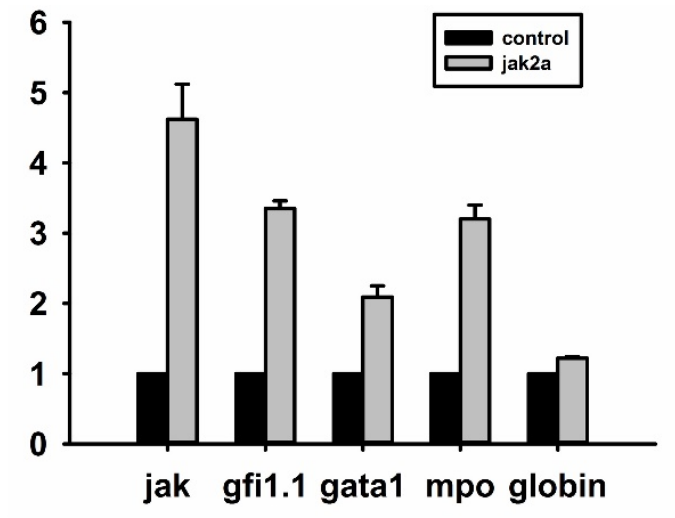

\begin{tabular}{lccc}
\hline Gene & Control & Jak2a mRNA & $P$ Value \\
\hline Jak2a & 1.00 & $4.62 \pm 0.50$ & $\mathrm{P}<0.05$ \\
Gfil.1 & 1.00 & $3.35 \pm 0.11$ & $\mathrm{P}<0.05$ \\
Gata 1 & 1.00 & $2.09 \pm 0.16$ & $\mathrm{P}<0.05$ \\
Mpo & 1.00 & $3.32 \pm 0.20$ & $\mathrm{P}<0.05$ \\
$\beta$-globin & 1.00 & $1.22 \pm 0.02$ & $\mathrm{P}<0.05$ \\
\hline
\end{tabular}

Figure 4. Examination of jak2a, gfil.1, gatal, mpo and hemoglobin mRNAs by real-time PCR in $1 \mathrm{dpf}$ embryos.

Con Jak2a-wt

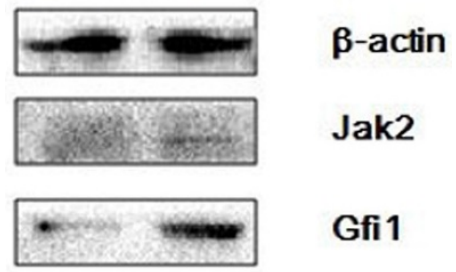

Figure 5. Western blot detection of jak2a and gfil expression in the overexpression and control embryos.

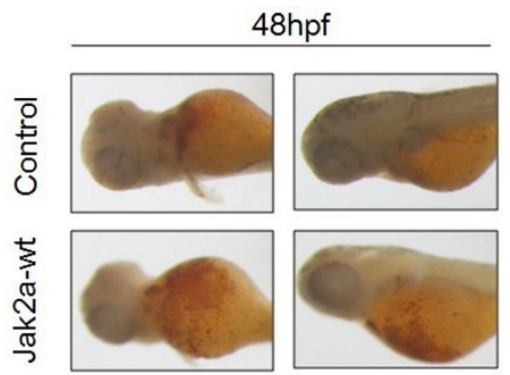

Figure 6. O-dianisidine staining of the control and jak2a-overexpressing embryos at 48 hpf.

\section{Effects of jak2a mRNA on erythropoiesis}

Previous studies have that the transient expression of jak2aV581F in zebrafish shares features of human PV in terms of increasing erythropoiesis and reducing erythropoietin at both the mRNA and protein levels. However, the development of myeloid hematopoiesis was not observed in the jak2av581F zebrafish model [16]. In our studies, to examine the function of the jak2a wild-type in hematopoiesis, we first examined the effects of jak $2 a$ on primitive hematopoiesis, which was estimated based on the presence of $\mathrm{Hb}$-staining with $\mathrm{O}$-dianisidine at $48 \mathrm{hpf}$. The O-dianisidine staining was positive in the ICM and yolk sac in both the control and jak2a groups. However, the staining was significantly increased in the ICM and yolk sac in the jak2a group (Figure 6). These findings were confirmed with the transgenic zebrafish lines Tg (gata1: gfp) and Tg (mpo: gfp). Jak2a was then injected into the embryos to create transgenic reporter fish expressing GFP under the gata1 and mpo promoters (Figure 7). In contrast to the control group, the jak2a overexpression group exhibited increased the numbers of $\mathrm{GFP}^{+}$cells in the gata1 embryos at $48 \mathrm{hpf}$. Quantitative analyses of the $\mathrm{GFP}^{+}$cells in the Tg (gata1: $g f p$ )-48-hpf embryos revealed that jak2a induced a significant increase in these cells in the Tg (gata1: $g f p$ ) embryos (uninjected embryos: $17.22 \pm 1.70 \%$; embryos injected with jak2a mRNA: $21.31 \pm 2.11 \%, \mathrm{p}<0.01$ ).

To further confirm that the observation was not specific to the transgenic zebrafish lines, we performed in situ hybridization to assess the expression of the erythroid-specific embryonic gata1 gene at $36 \mathrm{hpf}$. Increases in red cell production in the ICM were observed in the jak2a embryos compared with the control group (Figure 8). Quantitative analyses of the observed changes in the gata1 and $\beta$-embryonic hemoglobin levels presented in Figure 4 via real-time PCR revealed that the expressions of gata1 and $\beta$-embryonic hemoglobin were much higher than those in the control group, particularly for gata1. The real-time PCR results were consistent with these findings.

\section{Effects of jak2a mRNA on myeloid hematopoiesis}

The activation of jak2aV581F has been demonstrated to mediate the upregulation of erythroid but not myeloid cells [16]. Additionally, the jak2aUTR embryos exhibited reduced early expression of hematopoietic specification genes ( $\mathrm{scl}$ and $\mathrm{lmo2}$ ) as well as erythroid (gata1, ahe1, and Bhe1) and myeloid (spil [early]) and mpo [late]) differentiation genes [9]. The results from the jak2aUTR embryos suggested that jak2a may play an important role in increasing myeloid development. To test this hypothesis, the transgenic zebrafish line $\operatorname{Tg}(m p o: g f p)$ was used to examine the effects on myeloid development that were mediated by the injection of jak2a mRNA. The injected embryos exhibited expansion of the $\mathrm{GFP}^{+}$ cells in the rostral ICM compared with the control group (Figure 7). Fluorescence-activated sorting of the GFP+ cells in the injected group revealed that the percentage of positive cells was approximately 8.0, whereas a significant reduction in the $\mathrm{GFP}^{+}$ population was observed in the control group 
(uninjected embryos: $3.86 \pm 1.94$; embryos injected with jak2a mRNA: $6.64 \pm 1.30 \%, p<0.01$; Figure 7 ). Moreover, the expression of mpo was significantly increased in the jak2a embryos as assessed by Q-RT-PCR (Figure 4). Furthermore, the expressions of mpo and gata1 were examined during embryonic development via whole-mount in situ hybridization, and upregulations of both mpo and gata1 in the expanded ICM and yolk sac were observed (Figure 8).

\section{Discussion}

A previous study demonstrated that jak2a, which is one of two jak2 genes that are expressed in the developing embryo and larva, is expressed in the erythropoietic system and plays an early role in primitive erythropoiesis in zebrafish [3]. Moreover,
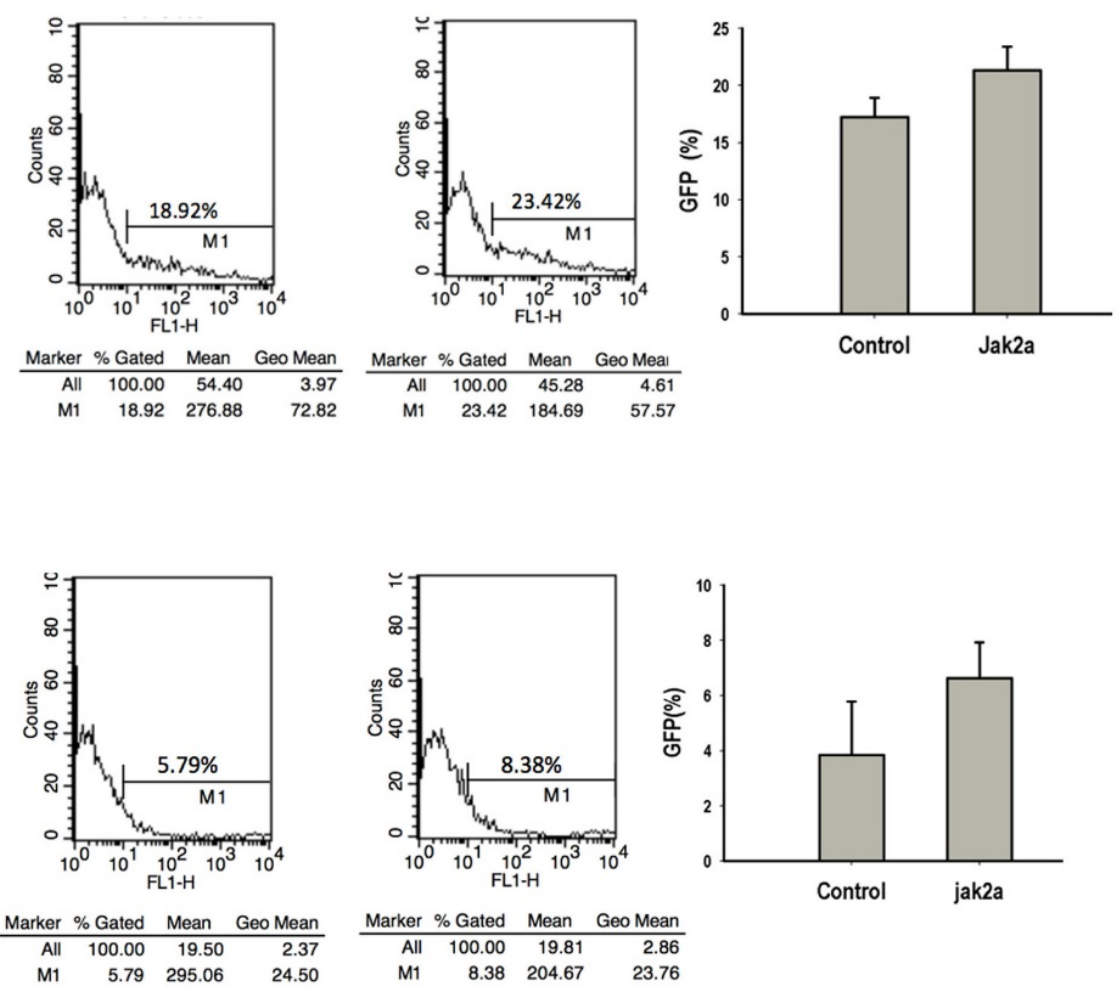

Figure 7. Flow cytometric analysis of effects of jak2a on erythropoiesis and myeloid hematopoiesis.

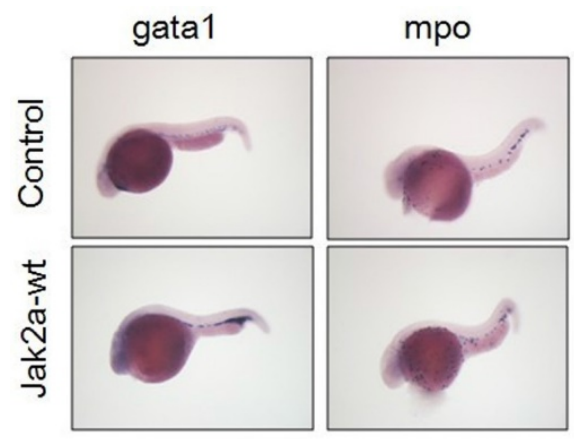

Figure 8. Whole-mount in situ hybridization was performed using gatal- and mpo-specific antisense RNA probes at $2 \mathrm{dpf}$ the jak2a message has been proven to play a role in the intermediate stage in the lineage of the primitive erythrocyte that occurs between the commitment of progenitors and the expression of the end-differentiated phenotype by whole-mount in situ hybridization [3]. In 3.5-dpf embryos, the expression of jak2a is not detectable by in situ hybridization, even though jak2a is indeed detected in the brain and eyes with whole-mount in situ hybridization [3]. However, the precise location of the jak2a transcript in adult zebrafish had not yet been determined. In this study, we first examined the timing and location of jak2a expression in adult tissues with real-time Q-PCR. Maternal transcripts of the zebrafish jak2a were detected at a low level at $0.5 \mathrm{hpf}$, and the peak in expression occurred at $3.75 \mathrm{hpf}$. However, in situ hybridization observations revealed that jak2a transcripts were first detected at a low level at $10 \mathrm{hpf}$ [3]. Although these results were inconsistent with our real-time Q-PCR analysis, we cannot exclude the possibility that jak2a transcripts are present in small amounts in the early stage of embryonic development. In adult zebrafish, jak2a was predominately expressed in the muscle, fin, skin, kidneys, cheek and gallbladder, and these findings are partially consistent with those of previous studies that have reported that the highest levels of expression occur in the skeletal muscle in humans [19]. Additionally, the kidneys, which are the primary hematopoietic organ from the larval stage to adulthood [20], exhibited high expression levels of jak2a in adult zebrafish, which indicates that jak2a may play a rather significant role in the adult zebrafish hematopoietic system.

Previous experimental studies have primarily used antisense morpholino oligonucleotides (MO) as the primary gene-specific knockdown approach to prove the role of jak2a during embryonic hematopoiesis [9]. In our study, we first used the pGM-T vector cloned with the full-length jak2a to encode the wild-type jak2a mRNA via in vitro transcription with the $T 7$ promoter following a strong RNA laddering pattern of approximately 
$3000 \mathrm{bp}$, which was detected via gel electrophoresis. Furthermore, the expression levels of the test and control groups were also proven by real-time Q-PCR and SDS-PAGE. In short, the gel electrophoresis and real-time Q-PCR results demonstrated that the process of the in vitro transcription of jak2a RNA was successful, and the injected jak2a RNA was rather stable in $1 \mathrm{dpf}$ zebrafish embryos. The Western blot results revealed that the T7 transcription system contained on the pGM-T vector can be used to prepare RNA substrates for studies of translation.

Jak2a $2581 F$, which is an ortholog of the human Jak2 ${ }^{V 617 F}$ mutation, was first discovered by the Anskar group in 2009. This group demonstrated that the zebrafish jak $2 a^{V 581 F}$ model shares many features with human PV because the model exhibits an increase in the expression of erythroid cells that is accompanied by a non-obvious expansion of the granulocytic and megakaryocytic lineages [16]. In this study, we mainly investigated the role of wild-type jak2a in embryonic hematopoiesis. The transient overexpression of jak2a in zebrafish embryos indeed increased erythroid hematopoiesis, as demonstrated by obvious increases in the expressions of the erythroid-specific embryonic gata1 gene and $\beta$-embryonic hemoglobin. Additionally, an upregulation of mpo gene expression was also observed. The GFP-positive cells in the transgenic zebrafish lines Tg (gata1: $g f p$ ) and $\operatorname{Tg}$ (mpo: $g f p$ ) were proven to be much more numerous in the jak2a overexpression group than in the control group. These results were further verified by whole-mount in situ hybridization. Moreover, the expression of zebrafish gfil.1 was increased to a rather high level. However, the precise mechanistic link between the increase in erythropoiesis and gfi1.1 was not further investigated in our experiment. Further investigations should determine whether the increase in jak2a is a form of positive feedback control that regulates the expression of gfi1.1. Additionally, a comparative study of the jak2a and jak2av581F overexpression zebrafish models should be undertaken to establish whether there are significant differences in embryonic hematopoiesis and especially erythropoiesis between these groups.

\section{Acknowledgments}

This study was supported by grant nos. 30971293 and 30670897 from the National Science Foundation of China, and the Natural Science Foundation of Hubei Provincial Department of Education (No. Q20152105).

\section{Competing Interests}

The authors have declared that no competing interest exists.

\section{References}

1. Baxter EJ, Scott LM, Campbell PJ, East C, Fourouclas N, Swanton S, et al. Acquired mutation of the tyrosine kinase JAK2 in human myeloproliferative disorders. Lancet. 2005; 365: 1054-61.

2. Kralovics R, Passamonti F, Buser AS, et al. A gain-of-function mutation of JAK2 in myeloproliferative disorders. New England Journal of Medicine. 2005; 352: 1779-90.

3. Levine RL, Wadleigh M, Cools J, Ebert BL, Wernig G, Huntly BJ, et al. Activating mutation in the tyrosine kinase JAK2 in polycythemia vera, essential thrombocythemia, and myeloid metaplasia with myelofibrosis. Cancer Cell. 2005; 7: 387-97.

4. Lippert E, Boissinot M, Kralovics R, Girodon F, Dobo I, Praloran V, et al. The JAK2-V617F mutation is frequently present at diagnosis in patients with essential thrombocythemia and polycythemia vera. Blood. 2006; 108: 1865-7.

5. Levine RL, Pardanani A, Tefferi A, Gilliland DG. Role of JAK2 in the pathogenesis and therapy of myeloproliferative disorders. Nature reviews Cancer. 2007; 7: 673-83

6. Campbell PJ, Baxter EJ, Beer PA, Scott LM, Bench AJ, Huntly BJ, et al. Mutation of JAK2 in the myeloproliferative disorders: timing, clonality studies, cytogenetic associations, and role in leukemic transformation. Blood. 2006; 108: 3548-55.

7. Veneri D, Capuzzo E, de Matteis G, Franchini M, Baritono E, Benati M, et al. Comparison of JAK2V617F mutation assessment employing different molecular diagnostic techniques. Blood Transfus. 2009; 7: 204-9.

8. Rane SG, Reddy EP. JAKs, STATs and Src kinases in hematopoiesis. Oncogene. 2002; 21: 3334-58.

9. Ma AC, Ward AC, Liang R, Leung AY. The role of jak2a in zebrafish hematopoiesis. Blood. 2007; 110: 1824-30.

10. Parganas E, Wang D, Stravopodis D, Topham DJ, Marine JC, Teglund S, et al. Jak2 is essential for signaling through a variety of cytokine receptors. Cell. 1998; 93: 385-95.

11. Neubauer $\mathrm{H}$, Cumano A, Muller $\mathrm{M}, \mathrm{Wu} \mathrm{H}$, Huffstadt U, Pfeffer K. Jak2 deficiency defines an essential developmental checkpoint in definitive hematopoiesis. Cell. 1998; 93: 397-409.

12. Weinstein BM, Schier AF, Abdelilah S, Malicki J, Solnica-Krezel L, Stemple DL, et al. Hematopoietic mutations in the zebrafish. Development. 1996; 123: 303-9.

13. Onnebo SM, Yoong SH, Ward AC. Harnessing zebrafish for the study of white blood cell development and its perturbation. Experimental hematology. 2004; 32: 789-96.

14. Oates AC, Brownlie A, Pratt SJ, Irvine DV, Liao EC, Paw BH, et al. Gene duplication of zebrafish JAK2 homologs is accompanied by divergent embryonic expression patterns: only jak2a is expressed during erythropoiesis. Blood. 1999; 94: 2622-36.

15. Leung AY, Mendenhall EM, Kwan TT, Liang R, Eckfeldt C, Chen E, et al. Characterization of expanded intermediate cell mass in zebrafish chordin morphant embryos. Developmental biology. 2005; 277: 235-54.

16. Ma AC, Fan A, Ward AC, Liongue C, Lewis RS, Cheng SH, et al. A novel zebrafish jak2a(V581F) model shared features of human JAK2(V617F) polycythemia vera. Experimental hematology. 2009; 37: 1379-86 e4.

17. Nilsson R, Schultz IJ, Pierce EL, Soltis KA, Naranuntarat A, Ward DM, et al. Discovery of genes essential for heme biosynthesis through large-scale gene expression analysis. Cell metabolism. 2009; 10: 119-30.

18. Amigo JD, Ackermann GE, Cope JJ, Yu M, Cooney JD, Ma D, et al. The role and regulation of friend of GATA-1 (FOG-1) during blood development in the zebrafish. Blood. 2009; 114: 4654-63.

19. Saltzman A, Stone M, Franks C, Searfoss G, Munro R, Jaye M, et al. Cloning and characterization of human Jak-2 kinase: high mRNA expression in immune cells and muscle tissue. Biochemical and biophysical research communications. 1998; 246: 627-33.

20. Carradice D, Lieschke GJ. Zebrafish in hematology: sushi or science? Blood. 2008; 111: 3331-42. 